\title{
On the Kirchhoff matrix, a new Kirchhoff index and the Kirchhoff energy
}

\author{
Ayse Dilek Maden ${ }^{1}$, Ahmet Sinan Cevik ${ }^{1 *}$, Ismail Naci Cangul ${ }^{2}$ and Kinkar C Das ${ }^{3}$
}

A retraction article was published for this article. It is available from the following link; http://www.journalofinequalitiesandapplications.com/content/2014/1/424.

"Correspondence:

sinan.cevik@selcuk.edu.tr

'Department of Mathematics,

Faculty of Science, Selçuk University,

Campus, Konya, 42075, Turkey

Full list of author information is

available at the end of the article

\begin{abstract}
The main purpose of this paper is to define and investigate the Kirchhoff matrix, a new Kirchhoff index, the Kirchhoff energy and the Kirchhoff Estrada index of a graph. In addition, we establish upper and lower bounds for these new indexes and energy. In the final section, we point out a new possible application area for graphs by considering this new Kirchhoff matrix. Since graph theoretical studies (including graph parameters) consist of some fixed point techniques, they have been applied in the fields such as chemistry (in the meaning of atoms, molecules, energy etc.) and engineering (in the meaning of signal processing etc.), game theory, and physics.
\end{abstract} MSC: 05C12; 05C50; 05C90

Keywords: Kirchhoff matrix; Kirchhoff Estrada index; Kirchhoff energy; lower and upper bounds 
more vertices, the matrix $L+\frac{1}{n} J$ is non-singular with the inverse

$$
X=\left\|x_{i j}\right\|=\left(L+\frac{1}{n} J\right)^{-1} .
$$

After that, the resistance distance $r_{i j}$ was defined in terms of $X$ as $r_{i j}=x_{i i}+x_{j j}-2 x_{i j}$ [1]. In addition to this last distance, the matrix whose $(i, j)$-entry is $r_{i j}$ was called the resistance distance matrix $R D=R D(G)$ which is symmetric and has a zero diagonal. As we mentioned previously, the concept of resistance distance has been studied a lot in chemical studies $[2,6]$. Furthermore, by considering the resistance distance of the graph $G$, the sum of resistance distance of all pairs of vertices as the equation

$$
K f(G)=\sum_{i<j} r_{i j}
$$

which was named the 'Kirchhoff index' [6, 7], was introduced and investigated.

\section{Kirchhoff matrix and Kirchhoff Laplacian matrix}

In the following, by considering the resistance distance between any two vertices, we first define the Kirchhoff matrix $K f_{A}(G)$ as a weighted adjacency matrix.

Let $G$ be an $(n, m)$-graph. Then $(i, j)$-entry of the $n \times n$ matrix $K f_{A}(G)$ is defined by

$$
k_{i j}= \begin{cases}r_{i j}, & \text { if } v_{i} \sim v_{j}, \\ 0, & \text { otherwise }\end{cases}
$$

We recall that the Laplacian matrix of the graph $G$ is $L(G)=D(G)-A(G)$, where $D(G)$ is the diagonal matrix of vertex degrees and $A(G)$ is the $(0,1)$-adjacency matrix of the graph $G$. Using (2) for the definition of $K f_{A}(G)$ and also using the Laplacian matrix, we can then define the Kirchhoff-Laplacian matrix $K f_{L}(G)$ of $G$ as

$$
K f_{L}(G)=K f_{D}(G)-K f_{A}(G)
$$

where $K f_{D}(G)=\operatorname{diag}\left(\sum_{j=1}^{n} k_{1 j}, \sum_{j=1}^{n} k_{2 j}, \ldots, \sum_{j=1}^{n} k_{n j}\right)$. For simplicity, let us label each $\sum_{j=1}^{n} k_{i j}$ by $k_{i}$. Then it is clear that

$$
K f_{D}(G)=\operatorname{diag}\left(k_{1}, k_{2}, \ldots, k_{n}\right) .
$$

The eigenvalues of $K f_{L}(G)$ are denoted by $\mu_{0}<\mu_{1}<\cdots<\mu_{b}$ such that the smallest eigenvalue is $\mu_{0}=0$ with eigenvector $j=(1,1, \ldots, 1)$. Since we have assumed that $G$ is connected, while the multiplicity of $\mu_{0}$ is one, multiplicities of the remaining eigenvalues can be denoted by $s_{0}=1, s_{1}, \ldots, s_{b}$.

After all, we can define a new Kirchhoff index in the form

$$
K f^{+}(G)=\sum_{i<j} k_{i j}
$$

where each $k_{i j}$ is as given in (2). 
By considering $K f_{L}(G)$, it is actually easy to rewrite the new Kirchhoff index, defined in (3), as a new form. In fact, this shows that $K f^{+}(G)$ is completely determined by the Kirchhoff-Laplacian spectrum. In detail, by considering this new form of $K f^{+}(G)$ (see (4) below), the ordering among the eigenvalues $\mu_{l}(1 \leq l \leq b)$ of $K f_{L}(G)$ and the equality

$$
2 K f^{+}(G)=\operatorname{Tr}\left(K f_{L}(G)\right)=\sum_{l=1}^{b} s_{l} \mu_{l},
$$

we can obtain a new lower and upper bound (see (5)) for this new Kirchhoff index as in the following proposition.

Proposition 1 For an $(n, m)$ graph $G$, let $\operatorname{Spec}\left(K f_{L}(G)\right)$ be the Kirchhoff-Laplacian spectrum of $G$, defined by

$$
\operatorname{Spec}\left(K f_{L}(G)\right)=\left\{\mu_{0}^{1}, \mu_{1}^{s_{1}}, \ldots, \mu_{b}^{s_{b}}\right\}
$$

Then a new Kirchhoff index of $G$ is defined by

$$
K f^{+}(G)=\frac{1}{2} \sum_{l=1}^{b} s_{l} \mu_{l}
$$

and bounded by

$$
\frac{(n-1)}{2} \mu_{1} \leq K f^{+}(G) \leq \frac{(n-1)}{2} \mu_{b}
$$

\section{On the Kirchhoff energy of a graph}

It is known that there are quite wide applications based on eigenvalues of the adjacency matrix in chemistry $[8,9]$. In fact one of the chemically (and also mathematically) most interesting graph-spectrum, based on quantities in the graph energy, is defined as follows.

Let $G$ be an $(n, m)$-graph and let $A(G)$ be its adjacency matrix having eigenvalues $\lambda_{1}, \lambda_{2}, \ldots, \lambda_{n}$. We note that by [10] these $\lambda$ 's are said to be the eigenvalues of the graph $G$ and to form its spectrum. Then the energy $E(G)$ of $G$ is defined as the sum of the absolute values of these eigenvalues as

$$
E=E(G)=\sum_{i=1}^{n}\left|\lambda_{i}\right| .
$$

We may refer to [11-13] for more details and new constructions on the graph energy. In view of evident success of the concept of graph energy, and because of the rapid decrease of open mathematical problems in its theory, energies based on the eigenvalues of other graph matrices have been introduced very widely. Among them the Laplacian energy $L E(G)$, pertaining to the Laplacian matrix, can be thought of as the first [14]. We note that the theory of energy-like graph invariants was firstly introduced by Consonni and Todeschini in [15]. Later on, Nikiforov [16] extended the definition of energy to arbitrary matrices making thus possible to conceive the incidence energy [17] based on the incidence matrix, etc. 
As in other energies mentioned in the above paragraph, we can define a new energy by considering the Kirchhoff matrix given in (2) as follows.

If $G$ is an $(n, m)$-graph, then the Kirchhoff energy of $G$, denoted by $E K f(G)$, is equal to

$$
E K f(G)=\sum_{i=1}^{n}\left|\delta_{i}\right|
$$

where each $\delta_{i}$ (with ordering $\delta_{1} \geq \delta_{2} \geq \cdots \geq \delta_{n}$ ) denotes the eigenvalues of the Kirchhoff matrix $K f_{A}(G)$. Basically, these eigenvalues are said to be the $K$-eigenvalues of $G$.

\subsection{Bounds for the Kirchhoff energy}

In this subsection we mainly present upper and lower bounds over the Kirchhoff energy defined in (6).

The first result is the following.

Theorem 1 Let $G$ be a graph with $n \geq 2$ vertices. Then

$$
E K f(G) \leq \sqrt{n \kappa},
$$

where $\kappa$ is the sum of the squares of entries of the Kirchhoff matrix $K f_{A}(G)$.

Proof We have that

$$
\operatorname{EKf}(G)=\sum_{i=1}^{n}\left|\delta_{i}\right| \text { and } \sum_{i=1}^{n} \delta_{i}^{2}=\kappa=2 \sum_{1 \leq i<j \leq n}\left(k_{i j}\right)^{2} .
$$

By the Cauchy-Schwartz inequality, we get

$$
\begin{aligned}
E K f(G) & \leq\left|\delta_{1}\right|+\left|\delta_{n}\right|+\sqrt{(n-2) \sum_{i=2}^{n-1} \delta_{i}^{2}} \\
& =\left|\delta_{1}\right|+\left|\delta_{n}\right|+\sqrt{(n-2)\left(\kappa-\delta_{1}^{2}-\delta_{n}^{2}\right)} .
\end{aligned}
$$

Consider the function

$$
f(x, y)=x+y+\sqrt{(n-2)\left(\kappa-x^{2}-y^{2}\right)}, \quad \text { where } x>0, y>0 .
$$

Now our aim is to find the maximum value of $f(x, y)$. To do that, we need to calculate the derivatives

$$
\begin{aligned}
& f_{x}=1-\frac{x \sqrt{n-2}}{\sqrt{\kappa-x^{2}-y^{2}}}, \quad f_{y}=1-\frac{y \sqrt{n-2}}{\sqrt{\kappa-x^{2}-y^{2}}}, \quad f_{x x}=-\frac{\left(\kappa-y^{2}\right) \sqrt{n-2}}{\left(\kappa-x^{2}-y^{2}\right)^{3 / 2}} \\
& f_{x y}=f_{y x}=-\frac{x y \sqrt{n-2}}{\left(\kappa-x^{2}-y^{2}\right)^{3 / 2}} \quad \text { and } \quad f_{y y}=-\frac{\left(\kappa-x^{2}\right) \sqrt{n-2}}{\left(\kappa-x^{2}-y^{2}\right)^{3 / 2}} .
\end{aligned}
$$


By a simple calculation, it is clear that the equality $f_{x}=f_{y}=0$ implies $x=y=\sqrt{\frac{\kappa}{n}}$ and then, for this equality between $x$ and $y$, it is also true that

$$
f_{x x}<0 \quad \text { and } \quad f_{x x} f_{y y}-f_{x y}^{2}=\frac{(n-2)\left(\kappa-x^{2}-y^{2}\right)}{\left(\kappa-x^{2}-y^{2}\right)^{3}}>0 .
$$

The calculations above actually conclude that $f(x, y)$ has a maximum value at $x=y=\sqrt{\frac{\kappa}{n}}$ and the required maximum value of this function is

$$
2 \sqrt{\frac{\kappa}{n}}+\sqrt{(n-2)\left(\kappa-2 \frac{\kappa}{n}\right)}=\sqrt{n \kappa}
$$

Hence the result.

The following lemma is needed for our other results that are given in this paper.

Lemma 1 Let $G$ be a connected $(n, m)$-graph and let $\delta_{1}, \delta_{2}, \ldots, \delta_{n}$ be the $K$-eigenvalues of $G$.

Then

$$
\sum_{i=1}^{n} \delta_{i}=0
$$

and

$$
\sum_{i=1}^{n} \delta_{i}^{2}=2 \sum_{1 \leq i<j \leq n}\left(k_{i j}\right)^{2}
$$

Proof We clearly have $\sum_{i=1}^{n} \delta_{i}=\operatorname{Tr}\left[K f_{A}(G)\right]=\sum_{i=1}^{n} k_{i i}=0$. Moreover, for $i=1,2, \ldots, n$, the $(i, i)$-entry of $\left[K f_{A}(G)\right]^{2}$ is equal to $\sum_{j=1}^{n} k_{i j} k_{j i}=\sum_{j=1}^{n}\left(k_{i j}\right)^{2}$. Hence, we obtain

$$
\sum_{i=1}^{n} \delta_{i}^{2}=\operatorname{Tr}\left[K f_{A}(G)\right]^{2}=\sum_{i=1}^{n} \sum_{j=1}^{n}\left(k_{i j}\right)^{2}=2 \sum_{1 \leq i<j \leq n}\left(k_{i j}\right)^{2},
$$

as required.

Theorem 2 If $G$ is a connected $(n, m)$-graph, then

$$
\sqrt{2 \sum_{1 \leq i<j \leq n}\left(k_{i j}\right)^{2}} \leq E K f(G) \leq \sqrt{2 n \sum_{1 \leq i<j \leq n}\left(k_{i j}\right)^{2}} .
$$

Proof In the Cauchy-Schwartz inequality $\left(\sum_{i=1}^{n} a_{i} b_{i}\right)^{2} \leq\left(\sum_{i=1}^{n} a_{i}^{2}\right)\left(\sum_{i=1}^{n} b_{i}^{2}\right)$, if we choose $a_{i}=1$ and $b_{i}=\left|\delta_{i}\right|$, then we get

$$
\left(\sum_{i=1}^{n}\left|\delta_{i}\right|\right)^{2} \leq n \sum_{i=1}^{n} \delta_{i}^{2}
$$

from which

$$
E K f(G)^{2} \leq 2 n \sum_{1 \leq i<j \leq n}\left(k_{i j}\right)^{2}
$$


Therefore this gives the upper bound for $\operatorname{EKf}(G)$.

Now, for the lower bound of $E K f(G)$, we can easily obtain the inequality

$$
\operatorname{EKf}(G)^{2}=\left(\sum_{i=1}^{n}\left|\delta_{i}\right|\right)^{2} \geq \sum_{i=1}^{n}\left|\delta_{i}\right|^{2}=2 \sum_{1 \leq i<j \leq n}\left(k_{i j}\right)^{2},
$$

which gives directly the required lower bound.

We should note that there is the second way to prove the upper bound that can be presented as follows.

Let us consider the sum

$$
M=\sum_{i=1}^{n} \sum_{j=1}^{n}\left(\left|\delta_{i}\right|-\left|\delta_{j}\right|\right)^{2}
$$

By a direct calculation, we obtain

$$
M=2 n \sum_{i=1}^{n}\left|\delta_{i}\right|^{2}-2\left(\sum_{i=1}^{n}\left|\delta_{i}\right| \sum_{j=1}^{n}\left|\delta_{j}\right|\right) .
$$

It follows from (7) and the definition of $\operatorname{EKf}(G)$ that

$$
M=4 n \sum_{1 \leq i<j \leq n}\left(k_{i j}\right)^{2}-2 E K f(G)^{2} .
$$

Here, since $M \geq 0$, we have $E K f(G) \leq \sqrt{2 n \sum_{1 \leq i<j \leq n}\left(k_{i j}\right)^{2}}$.

Hence the result.

In the following, we present a new lower bound which is better than the lower bound given in Theorem 2 .

Theorem 3 Let $G$ be a connected $(n, m)$-graph and let $\nabla$ be the absolute value of the determinant of the Kirchhoff matrix $\mathrm{Kf}_{A}(G)$. Then

$$
\sqrt{2 \sum_{1 \leq i<j \leq n}\left(k_{i j}\right)^{2}+n(n-1) \nabla^{2 / n}} \leq E K f(G)
$$

Proof In the light of Theorem 2, if we show the validity of the lower bound, then this finishes the proof.

By the definition of Kirchhoff energy given in (6) and by the equality in (7), we have

$$
\begin{aligned}
{[E K f(G)]^{2} } & =\left(\sum_{i=1}^{n}\left|\delta_{i}\right|\right)^{2}=\sum_{i=1}^{n}\left|\delta_{i}\right|^{2}+2 \sum_{1 \leq i<j \leq n}\left|\delta_{i}\right|\left|\delta_{j}\right| \\
& =2 \sum_{1 \leq i<j \leq n}\left(k_{i j}\right)^{2}+2 \sum_{1 \leq i<j \leq n}\left|\delta_{i}\right|\left|\delta_{j}\right| \\
& =2 \sum_{1 \leq i<j \leq n}\left(k_{i j}\right)^{2}+\sum_{i \neq j}\left|\delta_{i}\right|\left|\delta_{j}\right| .
\end{aligned}
$$


Since, for nonnegative values, the arithmetic mean is not smaller than the geometric mean, we have

$$
\begin{aligned}
\frac{1}{n(n-1)} \sum_{i \neq j}\left|\delta_{i}\right|\left|\delta_{j}\right| & \geq\left(\prod_{i \neq j}\left|\delta_{i}\right|\left|\delta_{j}\right|\right)^{1 / n(n-1)}=\left(\prod_{i=1}^{n}\left|\delta_{i}\right|^{2(n-1)}\right)^{1 / n(n-1)} \\
& =\prod_{i=1}^{n}\left|\delta_{i}\right|^{2 / n}=\nabla^{2 / n} .
\end{aligned}
$$

After that, by combining Equations (8) and (9), we obtain the required lower bound.

Theorem 4 If $G$ is a connected $(n, m)$-graph, then

$$
E K f(G) \leq \frac{2}{n} \sum_{1 \leq i<j \leq n}\left(k_{i j}\right)^{2}+\sqrt{(n-1)\left[2 \sum_{1 \leq i<j \leq n}\left(k_{i j}\right)^{2}-\left(\frac{2}{n} \sum_{1 \leq i<j \leq n}\left(k_{i j}\right)^{2}\right)^{2}\right]} .
$$

Proof We apply the standard procedure (see, for instance, $[18,19])$ to obtain such upper bounds.

By applying the Cauchy-Schwartz inequality to the two $(n-1)$ vectors $(1,1, \ldots, 1)$ and $\left(\left|\delta_{2}\right|,\left|\delta_{2}\right|, \ldots,\left|\delta_{n}\right|\right)$, where each $\delta_{i}(2 \leq i \leq n)$ is a $K$-eigenvalue, we have

$$
\begin{aligned}
& \left(\sum_{i=2}^{n}\left|\delta_{i}\right|\right)^{2} \leq(n-1)\left(\sum_{i=2}^{n}\left|\delta_{i}\right|^{2}\right), \\
& \left(E K f(G)-\delta_{1}\right)^{2} \leq(n-1)\left(2 \sum_{1 \leq i<j \leq n}\left(k_{i j}\right)^{2}-\delta_{1}^{2}\right), \\
& E K f(G) \leq \delta_{1}+\sqrt{(n-1)\left(2 \sum_{1 \leq i<j \leq n}\left(k_{i j}\right)^{2}-\delta_{1}^{2}\right)} .
\end{aligned}
$$

Now, let us consider the function

$$
f(x)=x+\sqrt{(n-1)\left(2 \sum_{1 \leq i<j \leq n}\left(k_{i j}\right)^{2}-x^{2}\right)} .
$$

In fact, by keeping in mind $\delta_{1} \geq 1$, we set $\delta_{1}=x$. Using

$$
\sum_{i=1}^{n} \delta_{i}^{2}=2 \sum_{1 \leq i<j \leq n}\left(k_{i j}\right)^{2}
$$

we get that

$$
x^{2}=\delta_{1}^{2} \leq 2 \sum_{1 \leq i<j \leq n}\left(k_{i j}\right)^{2} .
$$


In other words, $x \leq \sqrt{2 \sum_{1 \leq i<j \leq n}\left(k_{i j}\right)^{2}}$. Meanwhile, $f^{\prime}(x)=0$ implies that

$$
x=\sqrt{\frac{2}{n} \sum_{1 \leq i<j \leq n}\left(k_{i j}\right)^{2}} .
$$

Therefore $f$ is a decreasing function in the interval

$$
\sqrt{\frac{2}{n} \sum_{1 \leq i<j \leq n}\left(k_{i j}\right)^{2}} \leq x \leq \sqrt{2 \sum_{1 \leq i<j \leq n}\left(k_{i j}\right)^{2}}
$$

and

$$
\sqrt{\frac{2}{n} \sum_{1 \leq i<j \leq n}\left(k_{i j}\right)^{2}} \leq \frac{2}{n} \sum_{1 \leq i<j \leq n}\left(k_{i j}\right)^{2} \leq \delta_{1} .
$$

Hence,

$$
f\left(\delta_{1}\right) \leq f\left(\frac{2}{n} \sum_{1 \leq i<j \leq n}\left(k_{i j}\right)^{2}\right)
$$

and so the inequality in (10) holds.

\section{Kirchhoff Estrada index of graphs}

As a new direction for studying indexes and their bounds, we introduce Kirchhoff Estrada index and then investigate its bounds. Moreover, we obtain upper bounds for this new index involving the Kirchhoff energy of graphs. In order to do that, we divide this section into two cases.

We first recall that the Estrada index of a graph $G$ is defined by

$$
E E(G)=\sum_{i=1}^{n} e^{\lambda_{i}}
$$

where $\lambda_{1} \geq \lambda_{2} \geq \cdots \geq \lambda_{n}$ are the eigenvalues of the adjacency matrix $A(G)$ of $G$ (see [11, 20-26]). Denoting by $M_{k}=M_{k}(G)$ the $k$ th moment of the graph $G$, we get

$$
M_{k}=M_{k}(G)=\sum_{i=1}^{n}\left(\lambda_{i}\right)^{k},
$$

and recalling the power-series expansion of $e^{x}$, we have

$$
E E(G)=\sum_{k=0}^{\infty} \frac{M_{k}}{k !}
$$

By [23], it is well known that $M_{k}(G)$ is equal to the number of closed walks of length $k$ of the graph $G$. In fact the Estrada index of graphs has an important role in chemistry and physics, and there exists a vast literature that studied the Estrada index. In addition to 
Estrada's papers depicted above, we may also refer the reader to $[27,28]$ for more detailed information such as lower and upper bounds for $E E(G)$ in terms of the number of vertices and edges, and some inequalities between $E E(G)$ and $E(G)$.

\subsection{Bounds for the Kirchhoff Estrada index}

For an $(n, m)$-graph $G$, the definition of the Kirchhoff Estrada index $K E E(G)$ can be given as

$$
\operatorname{KEE}(G)=\sum_{i=1}^{n} e^{\delta_{i}}
$$

where $\delta_{1} \geq \delta_{2} \geq \cdots \geq \delta_{n}$ are the $K$-eigenvalues of $G$.

Let $L_{k}=L_{k}(G)=\sum_{i=1}^{n}\left(\delta_{i}\right)^{k}$. Then, similar to the $M_{k}$ case, the equality in (11) can be rewritten as

$$
\operatorname{KEE}(G)=\sum_{k=0}^{\infty} \frac{L_{k}}{k !}
$$

Thus the main result of the subsection is the following.

Theorem 5 Let $G$ be a connected $(n, m)$-graph. Then the Kirchhoff Estrada index is bounded as

$$
\sqrt{n^{2}+4 \sum_{1 \leq i<j \leq n}\left(k_{i j}\right)^{2}} \leq K E E(G) \leq n-1+e^{\sqrt{\sum_{1 \leq i<j \leq n}\left(k_{i j}\right)^{2}}} .
$$

Equality holds on both sides if and only if $G \approx K_{1}$.

Proof Lower bound. Directly from Equation (11), we get

$$
K E E^{2}(G)=\sum_{i=1}^{n} e^{2 \delta_{i}}+2 \sum_{1 \leq i<j \leq n} e^{\delta_{i} \delta_{j}} .
$$

By the arithmetic-geometric mean inequality $(A G M I)$, we also get

$$
\begin{aligned}
2 \sum_{1 \leq i<j \leq n} e^{\delta_{i} \delta_{j}} & \geq n(n-1)\left(\prod_{1 \leq i<j \leq n} e^{\delta_{i} \delta_{j}}\right)^{\frac{2}{n(n-1)}} \\
& =n(n-1)\left[\left(\prod_{i=1}^{n} e^{\delta_{i}}\right)^{n-1}\right]^{\frac{2}{n(n-1)}} \\
& =n(n-1)\left(e^{L_{1}}\right)^{\frac{2}{n}} \\
& =n(n-1) .
\end{aligned}
$$

By means of power-series expansion and $L_{0}=n, L_{1}=0, L_{2}=8 \sum_{1 \leq i<j \leq n}\left(k_{i j}\right)^{2}$, we clearly obtain

$$
\sum_{i=1}^{n} e^{2 \delta_{i}}=\sum_{i=1}^{n} \sum_{k \geq 0} \frac{\left(2 \delta_{i}\right)^{k}}{k !}=n+4 \sum_{1 \leq i<j \leq n}\left(k_{i j}\right)^{2}+\sum_{i=1}^{n} \sum_{k \geq 3} \frac{\left(2 \delta_{i}\right)^{k}}{k !} .
$$


Since we require a lower bound to be as good as possible, it looks reasonable to replace $\sum_{k \geq 3} \frac{\left(2 \delta_{i}\right)^{k}}{k !}$ by $4 \sum_{k \geq 3} \frac{\left(\delta_{i}\right)^{k}}{k !}$. Now, let us use a multiplier $t \in[0,4]$ instead of $4=2^{2}$. We then arrive at

$$
\begin{aligned}
\sum_{i=1}^{n} e^{2 \delta_{i}} & \geq n+4 \sum_{1 \leq i<j \leq n}\left(k_{i j}\right)^{2}+t \sum_{i=1}^{n} \sum_{k \geq 3} \frac{\left(\delta_{i}\right)^{k}}{k !} \\
& =n+4 \sum_{1 \leq i<j \leq n}\left(k_{i j}\right)^{2}-t \cdot n-t \sum_{1 \leq i<j \leq n}\left(k_{i j}\right)^{2}+t \sum_{i=1}^{n} \sum_{k \geq 0} \frac{\left(\delta_{i}\right)^{k}}{k !} \\
& =n \cdot(1-t)+(4-t) \sum_{1 \leq i<j \leq n}\left(k_{i j}\right)^{2}+t \cdot[\operatorname{KEE}(G)] .
\end{aligned}
$$

Now, for $n \geq 2$ and $m \geq 1$, it is easy to see that the function

$$
f(x)=\frac{x}{2}+\sqrt{\left(n-\frac{x}{2}\right)^{2}+(4-x) \sum_{1 \leq i<j \leq n}\left(k_{i j}\right)^{2}}
$$

monotonically increases in the interval $[0,4]$. As a result, the best lower bound for $K E E(G)$ is attained for $t=0$. This gives us the first part of the theorem.

Upper bound. By considering the definition and equality of $K E E(G)$, we clearly have

$$
\begin{aligned}
\operatorname{KEE}(G) & =n+\sum_{i=1}^{n} \sum_{k \geq 1} \frac{\left(\delta_{i}\right)^{k}}{k !}=n+\sum_{i=1}^{n} \sum_{k \geq 1} \frac{\left|\delta_{i}\right|^{k}}{k !} \\
& =n+\sum_{k \geq 1} \frac{1}{k !} \sum_{i=1}^{n}\left(\delta_{i}^{2}\right)^{\frac{k}{2}},
\end{aligned}
$$

and then

$$
\begin{aligned}
n+\sum_{k \geq 1} \frac{1}{k !} \sum_{i=1}^{n}\left(\delta_{i}^{2}\right)^{\frac{k}{2}} & \leq n+\sum_{k \geq 1} \frac{1}{k !}\left[\sum_{i=1}^{n}\left(\delta_{i}^{2}\right)\right]^{\frac{k}{2}} \\
& =n+\sum_{k \geq 1} \frac{1}{k !}\left[2 \sum_{1 \leq i<j \leq n}\left(k_{i j}\right)^{2}\right]^{\frac{k}{2}} \\
& =n-1+\sum_{k \geq 0} \frac{\left(\sqrt{2 \sum_{1 \leq i<j \leq n}\left(k_{i j}\right)^{2}}\right)^{k}}{k !} \\
& =n-1+e^{\sqrt{2 \sum_{1 \leq i<j \leq n}\left(k_{i j}\right)^{2}}} .
\end{aligned}
$$

Hence, we get the right-hand side of the inequality given in (12).

In addition to the above progress, it is clear that the equality in (12) holds if and only if the graph $G$ has all zero Kirchhoff eigenvalues. Since $G$ is a connected graph, this only happens when $G \approx K_{1}$.

Hence the result.

In [29], by considering the maximum eigenvalue, Zhou et al. presented a lower bound for the reciprocal distance matrix in terms of the sum of the $i$ th row of it. By the same idea, 
one can also give a lower bound for the maximum eigenvalue $\delta_{1}(G)$ in terms of the sum of the $i$ th row of the Kirchhoff matrix $K f_{A}(G)$ and for the number of vertices $n$. We should note that the proof of the following lemma can be done quite similarly as the proof of the related result in [29]. (At this point we recall that for simplicity, each $\sum_{j=1}^{n} k_{i j}$ was labeled by $k_{i}$ in Section 2.)

Lemma 2 Let $G$ be a connected graph with $n \geq 2$ vertices. Then

$$
\sqrt{\frac{\sum_{i=1}^{n} k_{i}^{2}}{n}} \leq \delta_{1}(G)
$$

where $k_{i}$ is the sum of the ith row of $K f_{A}(G)$. Here, the equality holds if and only if $k_{1}=k_{2}=$ $\cdots=k_{n}$.

Therefore the lower bound on the Kirchhoff Estrada index of the graph $G$ (which was one of our focusing points) can be given as the following theorem.

Theorem 6 Let $G$ be a connected $(n, m)$-graph with $n \geq 2$. Then we have

$$
K E E(G) \geq e^{\sqrt{\frac{\sum_{i=1}^{n} k_{i}^{2}}{n}}}+\frac{n-1}{e^{\frac{1}{n-1} \sqrt{\frac{\sum_{i=1}^{n} k_{i}^{2}}{n}}}} .
$$

In (13) the equality holds if and only if $G=K_{n}$.

Proof As a special case of the theory, if we assume that $G$ is a null graph $N_{n}$, then for each $1 \leq i \leq n$, we get $k_{i}=0$ and $\delta_{1}=\delta_{2}=\cdots=\delta_{n}=0$. Thus $\operatorname{KEE}(G)=n$ and equality holds in Equation (13). In the reverse part, if $K E E(G)=n$, then by $A G M I$, one can easily see that $\delta_{1}=\delta_{2}=\cdots=\delta_{n}=0$ and hence $G=N_{n}$.

As a general case, let us suppose that $G \neq N_{n}$. Therefore $\delta_{1}>0$. We then have

$$
\begin{aligned}
\operatorname{KEE}(G) & =e^{\delta_{1}}+e^{\delta_{2}}+\cdots+e^{\delta_{n}} \geq e^{\delta_{1}}+(n-1)\left(\prod_{i=2}^{n} e^{\delta_{i}}\right)^{\frac{1}{n-1}} \quad \text { by } A G M I \\
& \geq e^{\delta_{1}}+(n-1)\left(e^{-\delta_{1}}\right)^{\frac{1}{n-1}} \quad \text { since } \sum_{i=1}^{n} \delta_{i}=0 .
\end{aligned}
$$

Now, by considering the function $f(x)=e^{x}+\frac{n-1}{e^{\frac{x}{n-1}}}$ with its derivative $f^{\prime}(x)=e^{x}-e^{-\frac{x}{n-1}}$, where $x>0$, we easily conclude that $f$ is an increasing function for $x>0$. Hence, from (14) and by Lemma 2, we get

$$
K E E(G) \geq e^{\sqrt{\frac{\sum_{i=1}^{n} k_{i}^{2}}{n}}}+\frac{n-1}{e^{\frac{1}{n-1} \sqrt{\frac{\sum_{i=1}^{n} k_{i}^{2}}{n}}}} .
$$

This completes the proof of the inequality part of (13).

Now suppose that equality holds in (13). This implies that equalities also hold throughout (14)-(16). From the equality of (14) and by $A G M I$, we obtain $\delta_{2}=\delta_{3}=\cdots=\delta_{n}$. Since 
$\delta_{1}>0$ and $\sum_{i=1}^{n} \delta_{i}=0$, we must have $\delta_{2}<0$. Thus $G$ is a connected graph. Moreover, from the equality of (16), we have $\delta_{1}=k_{1}=k_{2}=\cdots=k_{n}$. Since $\delta_{2}=\delta_{3}=\cdots=\delta_{n}$ and $\delta_{1}=k_{i}$, by Lemma 2, $G$ is a complete graph $K_{n}$.

The converse part is clear, i.e., the equality holds in (13) for the complete graph $K_{n}$.

Hence the result.

\subsection{An upper bound for the Kirchhoff Estrada index involving the Kirchhoff energy}

Here, for a connected graph $G$, the main aim is to show that there exist two upper bounds for the Kirchhoff Estrada index $K E E(G)$ with respect to the Kirchhoff energy $E K f(G)$.

Theorem 7 Let $\mathrm{G}$ be as above. Then

$$
K E E(G)-E K f(G) \leq n-1-\sqrt{2 \sum_{1 \leq i<j \leq n}\left(k_{i j}\right)^{2}}+e^{\sqrt{2 \sum_{1 \leq i<j \leq n}\left(k_{i j}\right)^{2}}}
$$

and

$$
K E E(G) \leq n-1+e^{E K f(G)} .
$$

Equality holds in (17) or (18) if and only if $G \approx K_{1}$.

Proof By considering the proof of Theorem 5, we have

$$
\operatorname{KEE}(G)=n+\sum_{i=1}^{n} \sum_{k \geq 1} \frac{\left(\delta_{i}\right)^{k}}{k !} \leq n+\sum_{i=1}^{n} \sum_{k \geq 1} \frac{\left|\delta_{i}\right|^{k}}{k !} .
$$

Moreover, by considering the Kirchhoff energy defined in (6), we get

$$
K E E(G) \leq n+E K f(G)+\sum_{i=1}^{n} \sum_{k \geq 2} \frac{\left|\delta_{i}\right|^{k}}{k !}
$$

which leads to (as in Theorem 5)

$$
\begin{aligned}
K E E(G)-E K f(G) & \leq n+\sum_{i=1}^{n} \sum_{k \geq 2} \frac{\left|\delta_{i}\right|^{k}}{k !} \\
& \leq n-1-\sqrt{2 \sum_{1 \leq i<j \leq n}\left(k_{i j}\right)^{2}}+e^{\sqrt{\sum_{1 \leq i<j \leq n}\left(k_{i j}\right)^{2}}} .
\end{aligned}
$$

Hence we obtain the inequality in (17).

Another approximation to obtain an upper bound related to the relationship between $K E E(G)$ and $E K(G)$ can be presented as follows

$$
\begin{aligned}
K E E(G) & \leq n+\sum_{i=1}^{n} \sum_{k \geq 1} \frac{\left|\delta_{i}\right|^{k}}{k !} \\
& \leq n+\sum_{k \geq 1} \frac{1}{k !}\left(\sum_{i=1}^{n}\left|\delta_{i}\right|^{k}\right)
\end{aligned}
$$




$$
\begin{aligned}
& =n+\sum_{k \geq 1} \frac{[E K f(G)]^{k}}{k !} \\
& =n-1+\sum_{k \geq 0} \frac{[E K f(G)]^{k}}{k !},
\end{aligned}
$$

which implies

$$
K E E(G) \leq n-1+e^{E K f(G)}
$$

as claimed in (18). By a similar idea as in the previous results, the equality holds in (17) and (18) if and only if $G \approx K_{1}$.

\section{Final remark}

As it has been mentioned in some parts of the previous sections, it is well known that some special type of matrices, indexes and energies obtained from graphs play an important role in applications, especially, in computer science, optimization and chemistry. This section is devoted to pointing out a possible new application area in spectral graph theory by considering the Kirchhoff matrix defined in this paper. Although the problem mentioned in the following paragraphs would seem easy for some of the researchers, we cannot prove it at the moment and believe that it would be kept as a future project.

In [30, p.537], the authors defined the Kirchhoffmatrix over a loopless connected digraph, say $D$. In fact, by using the same notation as in this reference, we can define it as a matrix $\mathbf{K}:=\mathbf{M}_{x}$ obtained from the incidence matrix $\mathbf{M}$ of $D$ by deleting the row $\mathbf{m}(x)$. After that, by an algebraic approximation over digraphs, it was depicted that $\mathbf{K}$ is a basis of the row space of $\mathbf{M}$ (such that each element in the basis set was called tension). According to Sections 7, 10 and 20 in [30], since there is a direct relationship between cycles and bonds in graphs and digraphs, and since tensions in a graph (or a digraph) are the linear combination of the tensions associated with their bonds, the authors produced the relationship between the Kirchhoff matrix over the digraph $D$ and electrical networks (in Section 20).

In Equation (2) of this paper, we have defined the new Kirchhoff matrix in spectral graph theory and, as far as we know, there is no such study about it in the literature. Therefore, by considering the facts and results given in the previous paragraph, one can try to investigate a similar approximation to the relationship between this new $\operatorname{Kirchhoff}$ matrix over $(n, m)$ graph $G$ and electrical networks.

Competing interests

The authors declare that they have no competing interests.

Authors' contributions

All authors completed the paper together. All authors read and approved the final manuscript.

\section{Author details}

${ }^{1}$ Department of Mathematics, Faculty of Science, Selçuk University, Campus, Konya, 42075, Turkey. ${ }^{2}$ Department of Mathematics, Faculty of Arts and Science, Uludag University, Gorukle Campus, Bursa, 16059, Turkey. ${ }^{3}$ Department of Mathematics, Sungkyunkwan University, Suwon, 440-746, Republic of Korea.

\section{Acknowledgements}

This work was presented in The International Conference on the Theory, Methods and Applications of Nonlinear Equations. 


\section{References}

1. Xiao, W, Gutman, I: Resistance distance and Laplacian spectrum. Theor. Chem. Acc. 110, 284-289 (2003)

2. Xiao, W, Gutman, I: On resistance matrices. MATCH Commun. Math. Comput. Chem. 49, 67-81 (2003)

3. Klein, DJ: Graph geometry, graph metrics \& Wiener. Fifty years of the Wiener index. MATCH Commun. Math. Comput. Chem. 35, 7-27 (1997)

4. Chen, H, Zhang, F: Resistance distance and the normalized Laplacian spectrum. Discrete Appl. Math. 155, 654-661 (2007)

5. Buckley, F, Harary, F: Distance in Graphs. Addision-Wesley, Redwood (1990)

6. Klein, DJ, Randić, M: Resistance distance, applied graph theory and discrete mathematics in chemistry (Saskatoon, SK, 1991). J. Math. Chem. 12(1-4), 81-95 (1993)

7. Güngör, AD, Cevik, AS, Das, KC: On the Kirchhoff index and the resistance-distance energy of a graph. MATCH Commun. Math. Comput. Chem. 67(2), 541-556 (2012)

8. Cvetković, D, Rowlinson, P, Simić, SK: Introduction to the Theory of Graph Spectra. Cambridge University Press, Cambridge (2010)

9. Graovac, A, Gutman, I, Trinajstić, N: Topological Approach to the Chemistry of Conjugated Molecules. Springer, Berlin (1977)

10. Cvetković, DM, Doob, M, Sachs, H: Spectra of Graphs - Theory and Application. Academic Press, New York (1980)

11. Güngör, AD, Cevik, AS: On the Harary energy and Harary Estrada index of a graph. MATCH Commun. Math. Comput. Chem. 64(1), 281-296 (2010)

12. Gutman, I: The energy of a graph. Ber. Math.-Stat. Sekt. Forsch. Graz 103, 1-22 (1978)

13. Gutman, I: The energy of a graph: old and new results. In: Betten, A, Kohnert, A, Laue, R, Wassermann, A (eds.) Algebraic Combinatorics and Applications, pp. 196-211. Springer, Berlin (2001)

14. Zhou, B, Gutman, I: On Laplacian energy of graphs. MATCH Commun. Math. Comput. Chem. 57, 211-220 (2007)

15. Consonni, $\vee$, Todeschini, R: New spectral indices for molecule description. MATCH Commun. Math. Comput. Chem. 60, 3-14 (2008)

16. Nikiforov, V: The energy of graphs and matrices. J. Math. Anal. Appl. 326, 1472-1475 (2007)

17. Jooyandeh, MR, Kiani, D, Mirzakkah, M: Incidence energy of a graph. MATCH Commun. Math. Comput. Chem. 62, 561-572 (2009)

18. Koolen, J, Moulton, V: Maximal energy of graphs. Adv. Appl. Math. 26, 47-52 (2001)

19. Koolen, J, Moulton, V: Maximal energy of bipartite graphs. Graphs Comb. 19, 131-135 (2003)

20. Estrada, E: Characterization of 3D molecular structure. Chem. Phys. Lett. 319, 713-718 (2000)

21. Estrada, E: Characterization of the folding degree of proteins. Bioinformatics 18, 697-704 (2002)

22. Estrada, E: Characterization of amino acid contribution to the folding degree of proteins. Proteins 54, $727-737$ (2004)

23. Estrada, E, Rodríguez-Velázguez, JA: Subgraph centrality in complex networks. Phys. Rev. E 71, 056103 (2005)

24. Estrada, E, Rodríquez-Velázquez, JA: Spectral measures of bipartivity in complex networks. Phys. Rev. E 72, 046105 (2005)

25. Estrada, E, Rodríguez-Velázguez, JA, Randić, M: Atomic branching in molecules. Int. J. Quant. Chem. 106, 823-832 (2006)

26. Güngör, AD, Bozkurt, SB: On the distance Estrada index of graphs. Hacet. J. Math. Stat. 38(3), 277-283 (2009)

27. Deng, H, Radenković, S, Gutman, S: The Estrada index. In: Cvetković, D, Gutman, I (eds.) Applications of Graph Spectra, pp. 123-140. Math. Inst., Belgrade (2009)

28. Peňa, JAD, Gutman, I, Rada, J: Estimating the Estrada index. Linear Algebra Appl. 427, 70-76 (2007)

29. Zhou, B, Trinajstic, N: Maximum eigenvalues of the reciprocal distance matrix and the reserve Wiener matrix. Int J. Quant. Chem. 108, 858-864 (2008)

30. Bondy, JA, Murty, USR: Graph Theory. Graduate Texts in Mathematics, vol. 244. Springer, New York (2008)

doi:10.1186/1029-242X-2013-337

Cite this article as: Maden et al.: Retracted: On the Kirchhoff matrix, a new Kirchhoff index and the Kirchhoff energy. Journal of Inequalities and Applications 2013 2013:337.

\section{Submit your manuscript to a SpringerOpen ${ }^{\circ}$ journal and benefit from:}

- Convenient online submission

- Rigorous peer review

- Immediate publication on acceptance

- Open access: articles freely available online

- High visibility within the field

- Retaining the copyright to your article 